INPLASY

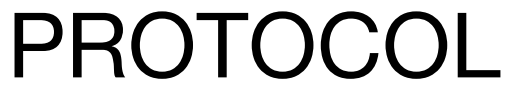

To cite: Guede-Rojas et al. Effectiveness of Exergames on rehabilitation outcomes in patients with osteoarthritis. A systematic review protocol. Inplasy protocol 202170072. doi:

10.37766/inplasy2021.7.0072

Received: 22 July 2021

Published: 23 July 2021

Corresponding author:

Francisco Guede-Rojas

francisco.guede@unab.cl

Author Affiliation:

Universidad Andres Bello.

Support: None.

Review Stage at time of this submission: The review has not yet started.

Conflicts of interest:

None declared.

\section{Effectiveness of Exergames on rehabilitation outcomes in patients with osteoarthritis. A systematic review protocol}

Guede-Rojas, F1; Aedo-Díaz, N2; Andrades-Torres, B3; González-Koppen, C4; Muñoz-Fuentes, M5; Carvajal-Parodi, C6; Mendoza-Sepúlveda, C7; Fuentes-Contreras, J8.

Review question / Objective: In patients with osteoarthritis, are exergames based on non-immersive virtual reality systems effective on clinical and psychosocial outcomes related to the rehabilitation process?

Condition being studied: $O A$ is a degenerative disease that affects the synovial joints characterized by the progressive deterioration of the hyaline cartilage, the subchondral bone, and the synovial membrane. Clinically, OA is accompanied by joint pain, crepitus, stiffness, and local inflammation. OA affects 302 million people in the world, being one of the main causes of disability. OA is also associated with reduced quality of life. The reduction of postural balance, muscle strength and proprioception increase the risk of falls in patients with $O A$, in addition, their levels of physical activity and social participation are also restricted. At the cognitive level, it is presumed that OA may be related to dementia, although the evidence is still scarce. Musculoskeletal pain in patients with $O A$ is associated with the deterioration of periarticular structures, however, it can also be recognized from the interaction of biological, psychological, and social factors related to loss of functionality. Better physical performance leads to decreased pain, increased functionality, social interaction, mental health, and quality of life.

INPLASY registration number: This protocol was registered with the International Platform of Registered Systematic Review and Meta-Analysis Protocols (INPLASY) on 23 July 2021 and was last updated on 23 July 2021 (registration number INPLASY202170072).

\section{INTRODUCTION}

Review question / Objective: In patients with osteoarthritis, are exergames based on non-immersive virtual reality systems effective on clinical and psychosocial outcomes related to the rehabilitation process? 
Rationale: Virtual reality (VR) has a progressive relevance in rehabilitation processes. VR is categorized as immersive, semi-immersive and non-immersive according to the sense of presence within the virtual environment. Non-immersive VR is characterized by a low level of immersion and its interaction through computer monitors and TV screens. Exergames are defined as video games that promote functional movement including activities of strength, balance, and flexibility, among others. Regarding the feasibility of exercise programs based on non-immersive VR, a recent study shows that they deliver a pleasant experience, increasing motivation and adherence. Regarding usability, they have an attractive, easy-to-use design that allows users to see their score and progress as they exercise. According to recent reviews, the effectiveness of exergames has been studied mainly in neurological conditions (for example, stroke, Parkinson's disease, multiple sclerosis, Alzheimer's disease, traumatic brain injury and myelopathy). However, in musculoskeletal conditions there is less information and only a recent systematic review analyzed the effectiveness of exergames in patients with knee/hip osteoarthritis (OA) and knee/hip arthroplasty, suggesting its benefits in several functional components. Therefore, it is necessary to carry out a systematic review focused exclusively on patients with $\mathrm{OA}$ in any joint complex.

Condition being studied: $O A$ is a degenerative disease that affects the synovial joints characterized by the progressive deterioration of the hyaline cartilage, the subchondral bone, and the synovial membrane. Clinically, $O A$ is accompanied by joint pain, crepitus, stiffness, and local inflammation. OA affects 302 million people in the world, being one of the main causes of disability. $O A$ is also associated with reduced quality of life. The reduction of postural balance, muscle strength and proprioception increase the risk of falls in patients with $O A$, in addition, their levels of physical activity and social participation are also restricted. At the cognitive level, it is presumed that $O A$ may be related to dementia, although the evidence is still scarce. Musculoskeletal pain in patients with $O A$ is associated with the deterioration of periarticular structures, however, it can also be recognized from the interaction of biological, psychological, and social factors related to loss of functionality. Better physical performance leads to decreased pain, increased functionality, social interaction, mental health, and quality of life.

\section{METHODS}

Search strategy: (1) Population: ((((Osteoarthritis, Hip[MeSH Terms]) OR (Osteoarthritis, Knee[MeSH Terms])) OR (Osteoarthritis, Spine[MeSH Terms])) OR (Osteoarthritis[MeSH Terms])) OR (Osteoarth*) (2) Intervention:

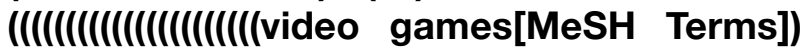
OR (virtual reality[MeSH Terms])) OR (exergam*)) OR (exergam*)) OR ("video game*")) OR (videogam*)) OR (video-gam*)) OR (video-based)) OR ("gaming technology")) OR (Wii)) OR (Nintendo)) OR (Kinect)) OR (Xbox)) OR (X-box)) OR (Eyetoy)) OR ("Play station")) OR (Play-station)) OR ("Serious gam*")) OR ("Dance dance revolution")) OR ("active video gam*")) OR (Non-immersive)) OR ("Non immersive")) OR (Nonimmersive).

Participant or population: Osteoarthritis patients.

Intervention: Exergames (non-immersive virtual reality).

Comparator: Control group: Any condition.

Study designs to be included: Randomized controlled trials (RCTs).

Eligibility criteria: (1) Inclusion criteria: Population: Adult patients diagnosed with $O A$ regardless of joint complex and degree of severity; Intervention: Exergames performed through any system or console based on non-immersive virtual reality; Comparator: Control group: No intervention, conventional physical therapy, or other therapeutic modalities; Outcomes: Measures of physical performance, 
cognitive performance, musculoskeletal pain, and psychosocial aspects; Time: Studies of the last $\mathbf{1 0}$ years; Type of study: Randomized controlled trials (RCTs); Setting: not restricted; Language: English. (2) Exclusion criteria: Unpublished studies, clinical trial protocols, secondary studies, and conference reports.

Information sources: Electronic searches will be carried out in the databases: Scopus, Web of Science, PubMed, and PEDro. In addition, a manual search from the reference list of the included studies.

Main outcome(s): Any specific measurement relative to: 1) Physical performance, 2) Cognitive performance, 3) Musculoskeletal pain, or 4) Psychosocial dimensions. The syntheses will be organized by each category of outcome. According to the types of variables the following measures of effect will be used: Difference of means/Standard difference of means (for numerical) or Difference of proportions/Relative risk/Odds ratio (for categorical).

Additional outcome(s): None.

Data management: (1) The results of the databases will be imported to the Rayyan platform. (2) After eliminating duplicates, two independent reviewers will manage the selection of studies. The concordance will be analysed using the kappa index. (3) The selection process will initially consider a screening for titles and abstracts, and then a comprehensive review of the full text. (4) Reviewers will independently extract information from included studies. (5) The data extraction form will include: Author; Year of publication; Primary objective; Setting; Population (sample size, distribution by sex, mean age, clinical condition, inclusion criteria); Intervention (experimental-control groups, technologysystems used, frequency); Measurement tools; Main results; Measures of effect magnitude or clinical significance; Adverse events; Feasibility and usability. (6) Possible conflicts at each stage of the study will be resolved by consensus.
Quality assessment / Risk of bias analysis: (1) The 11-point PEDro scale. For the assessment of the methodological quality of individual studies. (2) Cochrane Revman 5.4 Software. For the assessment of individual and overall risk-of-bias.

Strategy of data synthesis: According to the number of included studies their comparisons and their heterogeneity, the possibility of performing a meta-analysis using the Revman 5.4 software will be evaluated. Heterogeneity will be calculated using $\mathrm{I}^{2} \quad(0 \%$ to $40 \%$ might not be important; $30 \%$ to $60 \%$ may represent moderate heterogeneity; $50 \%$ to $90 \%$ may represent substantial heterogeneity; $75 \%$ to $100 \%$ considerable heterogeneity). The Chi-square test will be used to evaluate whether the differences observed in the results were compatible with chance $(p<0.05)$. If the heterogeneity is considerable a qualitative synthesis of the findings will be performed.

Subgroup analysis: A possible subgroup analysis may be carried out depending on the clinical characteristics of the participants, the types of intervention or the measurement methods.

Sensitivity analysis: To analyse the stability of the conclusions, studies with lower methodological quality and higher risk of bias will be excluded.

Language: English only.

Country(ies) involved: Chile.

Keywords: Osteoarthritis; Video games; Virtual reality; Rehabilitation; Psychosocial functioning.

Dissemination plans: Peer-reviewed indexed journal.

Contributions of each author:

Author 1 - Francisco Guede-Rojas - Study design, guidance and drafted the manuscript.

Email: francisco.guede@unab.cl

Author 2 - Natalia Aedo-Díaz - Literature search and selection. 
Email: nataliaedo.85@gmail.com

Author 3 - Barbara Andrades-Torres Literature search and selection.

Email: B.andradestorres@gmail.com

Author 4 - Constanza González-Koppen Data collection and synthesis.

Email: c.gonzlezkoppen@uandresbello.edu

Author 5 - Mirkko Muñoz-Fuentes - Data collection and synthesis.

Email: Mircko.-@hotmail.com

Author 6 - Claudio Carvajal-Parodi - Data analysis. Quality rating. Reviewing the manuscript.

Email: Claudio.Carvajal@uss.cl

Author 7 - Cristhian Mendoza-Sepúlveda Data analysis. Quality rating. Reviewing the manuscript.

Email: cristhian.mendoza@uss.cl

Author 8 - Jorge Fuentes-Contreras - Data analysis. Quality rating. Reviewing the manuscript.

Email: jorgef@ualberta.ca 